\title{
Les corps saints inhumés dans les sanctuaires du diocèse de Langres : conservation, exposition, vénération $\left(\mathrm{VI}^{\mathrm{e}}-\mathrm{XVI}^{\mathrm{e}}\right.$ siècle)
}

Thèse de doctorat de l'université de Bourgogne (ARTeHIS-UMR 5594), sous la direction de Vincent Tabbagh, Dijon, décembre 2007.

\section{Laurent Durnecker}

\section{(2) OpenEdition}

\section{Journals}

Édition électronique

URL : https://journals.openedition.org/cem/7632

DOI : $10.4000 /$ cem.7632

ISSN : 1954-3093

\section{Éditeur}

Centre d'études médiévales Saint-Germain d'Auxerre

Édition imprimée

Date de publication : 15 août 2008

ISSN : 1623-5770

\section{Référence électronique}

Laurent Durnecker, «Les corps saints inhumés dans les sanctuaires du diocèse de Langres

conservation, exposition, vénération ( $V\left|I^{e}-X V\right| e$ siècle) », Bulletin du centre d'études médiévales d'Auxerre I BUCEMA [En ligne], 12 | 2008, mis en ligne le 03 juillet 2008, consulté le 22 septembre 2022. URL : http://journals.openedition.org/cem/7632 ; DOI : https://doi.org/10.4000/cem.7632

Ce document a été généré automatiquement le 22 septembre 2022.

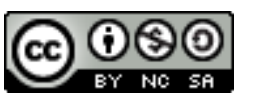

Creative Commons - Attribution - Pas d'Utilisation Commerciale - Partage dans les Mêmes Conditions 4.0 International - CC BY-NC-SA 4.0

https://creativecommons.org/licenses/by-nc-sa/4.0/ 


\section{Les corps saints inhumés dans les sanctuaires du diocèse de Langres : conservation, exposition, vénération $\left(\mathrm{VI}^{\mathrm{e}}-\mathrm{XVI}^{\mathrm{e}}\right.$ siècle)}

Thèse de doctorat de l'université de Bourgogne (ARTeHIS-UMR 5594), sous la direction de Vincent Tabbagh, Dijon, décembre 2007.

\section{Laurent Durnecker}

\section{Approche, démarche}

Depuis la thèse pionnière de Nicole Herrmann-Mascard, publiée en $1975^{1}$, le culte des reliques représente un champ fécond de l'historiographie. Embrassant aujourd'hui le vaste domaine des croyances et des pratiques de dévotion, il concerne également l'architecture religieuse, les manifestations artistiques et l'expression du pouvoir. En 1997, le colloque de Boulogne-sur-Mer a montré cette diversité ${ }^{2}$, dont témoigne également le volume de la revue PECIA paru en $2006^{3}$. L'étude présentée ici a pour principal objet une catégorie particulière de reliques: les corps saints. Le culte liturgique qui les entoure au Moyen Âge revêt plusieurs caractéristiques, dont la plus ancienne et la plus évidente est un rapport étroit au domaine funéraire. Il recouvre également certains rituels spécifiques tels que l'élévation, par laquelle la dépouille du saint est exhumée et placée dans un nouveau réceptacle, et le démembrement, c'est-àdire le prélèvement sur celle-ci d'un ou plusieurs ossements notables. L'objectif premier du doctorat a consisté à étudier ce processus d'élévation et de fragmentation sur la longue durée, sans se limiter à un «âge d'or " (pré)supposé ou à un seul type de sanctuaire. L'enquête a porté sur l'ancien diocèse de Langres, l'un des plus vastes du royaume de France avant la création de l'évêché de Dijon en 1731. Il faisait partie des vingt-quatre évêchés primitifs de la Gaule. Cette terre de confins, située à la fin du Moyen Âge aux portes de l'Empire, a accueilli d'importantes fondations monastiques, dont les plus connues sont les monastères de Réôme ( $V^{\mathrm{e}}$ siècle), Molesmes (1075) et Clairvaux (1115), la troisième des quatre abbayes filles de Cîteaux. Plus bourguignon 
que champenois, le diocèse de Langres apparait également marqué par l'importance de Dijon, où les évêques résident de la fin du Ve à la fin du VIe siècle au plus tôt. C'est sous les murs de ce castrum que se développe au $\mathrm{VI}^{\mathrm{e}}$ siècle le culte de saint Bénigne, apôtre de la Bourgogne, sur le tombeau duquel est bâtie une basilique desservie au VIII ${ }^{\mathrm{e}}$ siècle par des moines.

2 S'il semblait évident de commencer l'étude par les "origines ", le XVI siècle ne s'est imposé que progressivement comme limite basse. Dans le diocèse de Langres comme ailleurs, la vie religieuse connaît alors d'importants changements, pour certains très brusques. Ainsi, les abbayes prises en compte ont pour la plupart été détruites pendant les guerres de Religion. De nombreux corps saints ont alors été profanés et les reliquaires médiévaux, détruits ou dépouillés. Ces événements ne doivent pas occulter une évolution plus profonde du culte des saints, perceptible localement dès les années 1520 et surtout 1530 .

Trois axes principaux sous-tendent le travail entrepris. Le premier a trait à l'évolution des modes de conservation des corps saints. Avant de rechercher les causes de leurs élévations, translations et démembrements successifs, il était indispensable d'en établir le plus précisément possible la chronologie, site par site, des origines au XVI ${ }^{\mathrm{e}}$ siècle. Ces différents événements devaient en outre être considérés à l'aune du contexte monumental, politique, voire également économique, propre à chacun des sanctuaires pris en compte. Ensuite, seulement, pouvaient être distinguées certaines périodes marquées, à l'échelle du diocèse, par des séries de translations et de démembrements ce qui supposait d'en rechercher les causes profondes. Cette seconde étape de l'étude a consisté à déterminer si l'évolution des modes de conservation et d'exposition des corps saints a suivi celle, plus générale, des pratiques de vénération. A-t-elle été seulement conditionnée par des besoins liturgiques, pratiques, ou a-t-elle également découlé de considérations théologiques et symboliques? Ce questionnement a intéressé non seulement le besoin de toucher ou de voir l'objet de la dévotion, mais aussi la signification de l'autel chrétien. L'autel et les reliques sont en effet intimement liés depuis les origines du culte des saints, à cause notamment du rapprochement entre le sacrifice du Christ et le sacrifice des martyrs.

Enfin, il s'est avéré indispensable de replacer l'histoire de la vénération des corps saints dans celle, plus large, des pèlerinages. Il convenait notamment de déterminer si ce type de reliques a été supplanté à la fin du Moyen Âge par d'autres supports de la dévotion comme les statues miraculeuses de la Vierge et les restes de saints universels. Établir la dynamique générale des pèlerinages dans le diocèse de Langres représentait un moyen de le savoir. Cette entreprise, combinée à l'analyse des livres liturgiques, a permis d'appréhender la place des saints locaux et en particulier diocésains après le XII ${ }^{\mathrm{e}}$ siècle. Elle s'est nourrie des discussions et réflexions méthodologiques menées depuis 2002 au sein du programme «Inventaire des sanctuaires et lieux de pèlerinage chrétiens en France", coordonné par Catherine Vincent et intégré au groupe de recherche "Sources, acteurs et lieux de la vie religieuse à l'époque médiévale » (GDR 2513 du CNRS) ${ }^{4}$.

Les sources

5 En ce qui concerne le territoire pris en compte, les trois principaux axes de recherche envisagés - conservation, exposition et vénération des corps saints - ont pu être explorés à la lumière d'une documentation abondante. Plusieurs cartulaires sont conservés à partir du XI ${ }^{\mathrm{e}}$ siècle; ceux de Saint-Bénigne et de Saint-Étienne de Dijon 
sont particulièrement bien tenus, de même que ceux de l'abbaye cistercienne de Clairvaux à partir du XII ${ }^{e}$ siècle. Ils comprennent de précieuses indications relatives, notamment, aux vocables d'églises ou d'autels, voire aux reliques elles-mêmes. Beaucoup plus riches et plus précises sont toutefois les sources narratives. Les plus connues des historiens sont assurément la Chronique de Saint-Bénigne de Dijon, rédigée vers 1055-1075, et dans une moindre mesure la Vie de Guillaume de Volpiano écrite par Raoul Glaber. La Chronique de Bèze et la Vie de Garnier de Mailly, rédigées au XII ${ }^{\mathrm{e}}$ siècle, restent, quant à elles, relativement peu citées, mais n'en demeurent pas moins précieuses pour l'histoire de la Bourgogne et, notamment, du culte des saints. La première des deux œuvres intéresse l'histoire de l'abbaye bénédictine Saint-Pierre-etSaint-Paul de Bèze. La seconde est un panégyrique consacré à Garnier, prévôt de SaintÉtienne de Dijon mort en 1053. Cette antique église de clercs fut érigée en abbaye de chanoines réguliers entre 1113 et 1125 .

6 Ce sont les vies de saints et les recueils de miracles qui représentent, et de loin, la masse documentaire la plus importante pour la période qui s'étend du IX ${ }^{\mathrm{e}}$ au XII ${ }^{\mathrm{e}}$ siècle. Posant de nombreux problèmes d'interprétation, voire également de datation, ils sont connus pour plusieurs d'entre eux à travers des manuscrits carolingiens. La production hagiographique atteint sans aucun doute son apogée au $\mathrm{X}^{\mathrm{e}}$ siècle et dans la première moitié du $\mathrm{XI}^{e}$ siècle. Elle diminue nettement après le milieu du XII ${ }^{e}$ siècle, restant cantonnée jusqu'à la fin du siècle suivant à l'abbaye de Molesmes et à la cathédrale de Langres, pour l'essentiel. Deux ensembles principaux peuvent être distingués à cette époque: les sources juridiques d'une part (cartulaires) et, de l'autre, les textes liturgiques - calendriers, martyrologes, ordinaires et coutumiers. À partir du XIII ${ }^{e}$ siècle, s'ajoutent les sources nécrologiques (obituaires et testaments) qui comprennent des renseignements précieux relatifs à l'instauration ou à l'augmentation de fêtes, voire également à des legs de reliquaires et d'autres objets.

7 La documentation apparait plus variée et dans l'ensemble abondante dans les deux derniers siècles du Moyen Âge, malgré plusieurs lacunes. En plus des testaments des ecclésiastiques de Langres et des sources liturgiques, bien conservés, le fonds du chapitre cathédral livre une importante série de comptes qui s'étend de la fin du XIII au premier tiers du XV $\mathrm{V}^{\mathrm{e}}$ siècle. Ils permettent d'appréhender, parfois avec précision, la fabrication de luminaire et l'évolution des fêtes. Toutefois, ces sources ne compensent que partiellement la mauvaise conservation des délibérations capitulaires antérieures à la fin $\mathrm{du} \mathrm{XV}^{\mathrm{e}}$ siècle. Le Moyen Âge n'a par ailleurs livré aucun inventaire de reliques détaillé en ce qui concerne la cathédrale. Dans les autres sanctuaires du diocèse, les plus anciens textes de ce type ne sont pas antérieurs à la charnière des $\mathrm{XIV}^{\mathrm{e}}$ et $\mathrm{XV}^{\mathrm{e}}$ siècles - Saint-Étienne de Dijon, Saint-Bénigne de Dijon et Clairvaux. Le faible intérêt des procès-verbaux de visites pastorales, très laconiques et conservés seulement à partir du dernier tiers $\mathrm{du} \mathrm{XV}^{\mathrm{e}}$ siècle, représente la dernière lacune importante de la documentation.

8 À partir du dernier tiers du XIV ${ }^{\mathrm{e}}$ siècle, celle-ci se caractérise par un déséquilibre flagrant entre le siège épiscopal et la capitale du duché de Bourgogne, qui a livré des sources très nombreuses et particulièrement bien conservées. Les protocoles de notaires comportent plusieurs mentions de pèlerinages et de legs, noyées dans une masse d'informations très variées. Beaucoup plus intéressants en la matière sont les comptes des ducs de Bourgogne, dans lesquels sont évoqués de nombreux pèlerinages et dons faits à différents sanctuaires. Les fonds des différentes églises dijonnaises, et 
notamment leurs comptes, permettent par ailleurs d'appréhender les aménagements intérieurs des édifices et les modes d'exposition des reliques.

Les différences qualitatives et quantitatives de la documentation relative au sujet d'étude, très nettes d'une période à l'autre, ne semblent pas résulter uniquement des aléas de la conservation. Elles traduisent aussi, dans une certaine mesure, une évolution des conceptions du clergé en ce qui concerne le culte des reliques et plus généralement leur utilisation. En la matière, trois phases ont pu être distinguées et correspondent au plan de la thèse.

Résultats

10 La première partie embrasse la longue période comprise entre le $\mathrm{VI}^{\mathrm{e}}$ et le premier tiers $\mathrm{du} \mathrm{XII}^{\mathrm{e}}$ siècle. Le sanctoral du diocèse se développe lentement; il est fixé presque définitivement au milieu du $\mathrm{XI}^{\mathrm{e}}$ siècle. Sa première caractéristique réside dans le très faible nombre des saints évêques: trois seulement vers l'an Mil (Didier, Grégoire et Urbain). La cité épiscopale s'efface derrière Dijon pendant le haut Moyen Âge, comme en témoigne l'annexion de la Passion des saints Jumeaux de Langres au cycle de saint Bénigne. Le sanctoral diocésain reste par ailleurs tout à fait traditionnel. À l'exception du martyr Gengoul, membre de l'aristocratie langroise assassiné au $\mathrm{VII}^{\mathrm{e}}$ ou au VIII siècle, tous les saints locaux sont liés aux premiers temps du christianisme, y compris ceux dont les noms n'apparaissent qu'au $\mathrm{X}^{\mathrm{e}}$ ou au $\mathrm{XI}^{\mathrm{e}}$ siècle, comme saint Vorles, prêtre de Marcenay. La volonté de se référer au passé se traduit par la conservation de la sépulture du saint, mais aussi par la construction de tombeaux d'aspect archaïque ; elle conduit également à l'édification de cryptes d'aspect ancien, comme par exemple celle de Saint-Geosmes (près de Langres). La cathédrale, paradoxalement, n'apparaît pas concernée par ce processus, mais voit privilégier durablement la sainteté universelle. Dédiée à partir du IX ${ }^{\mathrm{e}}$ et peut-être dès le VIII siècle à saint Mammès, illustre martyr de Césarée, elle se dote successivement de plusieurs de ses reliques, dont un bras dans les années 1070.

11 Parallèlement, l'époque carolingienne et les environs de l'an Mil se caractérisent par une réorganisation de la géographie sacrée du diocèse, d'une part, à travers les translations des dépouilles de plusieurs saints locaux et, de l'autre, par l'apport de corps saints provenant (ou supposés provenir) de contrées plus lointaines. Dans le diocèse de Langres, les invasions normandes n'ont eu qu'un impact réduit sur ce phénomène. Beaucoup plus importante a été la volonté, émanant de certains seigneurs, d'asseoir leurs possessions sur le plan spirituel. Enfin, la période comprise entre la fin du IX et le milieu du $\mathrm{XII}^{\mathrm{e}}$ siècle représente l'âge d'or des miracles, qui sont relatés parfois avec beaucoup de détails pour édifier, au moyen de l'exemple, les fidèles et les pèlerins mais aussi les clercs eux-mêmes.

12 Dès le milieu du XII ${ }^{e}$ siècle, à une époque caractérisée par le renforcement définitif de l'encadrement paroissial, les miracles ne représentent plus semble-t-il un outil d'édification des fidèles. Le clergé ne s'emploie guère à promouvoir de nouveaux cultes, exception faite des moines de Molesmes qui publient au début du XIII siècle les miracles accomplis par leur abbé Robert. La seule sainteté imitable reste celle de Bernard de Clairvaux, qui ne constitue réellement un modèle que pour les moines cisterciens eux-mêmes. À la fin du Moyen Âge, la "poursuite de la sainteté ", décrite par André Vauchez pour d'autres contrées, est nulle dans le diocèse de Langres comme d'ailleurs dans l'ensemble de la Bourgogne ; les ordres mendiants, très peu influents sur le plan local, n'ont pas infléchi cette tendance ${ }^{5}$. Les reliques conservent en revanche un 
rôle déterminant dans l'expression d'une autorité spirituelle et temporelle, parfois par leur seule présence. Le cas de Saint-Étienne de Dijon semble à cet égard très significatif. Il met en lumière une utilisation juridique des restes saints qui s'applique en l'occurrence à des reliques fragmentaires. Au XII ${ }^{\mathrm{e}}$ siècle, presque tous les saints patrons des églises placées sous la dépendance de cette abbaye sont représentés par des reliques enfermées dans les autels et en particulier le maître-autel de l'abbatiale : les possessions de Saint-Étienne y sont, pour ainsi dire, résumées, scellées. Dans la cité épiscopale c'est le crâne de saint Mammès, transféré de Constantinople après la IV ${ }^{e}$ croisade (1204) qui apparaît emblématique d'une autorité spirituelle. Assimilé explicitement au Christ, tête de l'Église, il est aussi présenté comme une allégorie de la cathédrale, tête de l'église diocésaine. Dans le même temps, les moines de SaintBénigne de Dijon privilégient l'utilisation liturgique des reliquaires ; l'ordinaire, type de texte destiné par nature au seul usage des religieux, témoigne d'une volonté de codifier et de hiérarchiser les différents honneurs dus à chaque saint. L'absence presque totale de Miracula après les années 1140 dénote un certain désintérêt des clercs pour les pèlerinages; malgré tout, elle ne saurait constituer la preuve absolue d'une désaffection des laïcs à leur égard. Des fondations de luminaires témoignent en effet, ici et là, de la vénération, au moins locale, qui entoure quelques corps saints, à SaintBénigne et Til-Châtel notamment.

À partir du milieu du XII ${ }^{e}$ siècle, les corps de plusieurs saints du diocèse sont transférés dans des châsses et fragmentés - saint Vorles et saint Bénigne par exemple -; leurs cultes restent malgré tout relativement effacés jusqu'à la fin du XIII ${ }^{\mathrm{e}}$ siècle. La situation change nettement à partir des années 1280 , évolution traitée dans la troisième et dernière partie.

14 Entre 1280 et 1330 principalement, plus localement jusque dans les années 1340, plusieurs corps saints sont transférés dans des châsses très richement ornées et placées en position éminente dans le chœur. Leurs chefs sont dans le même temps mis à part dans des chefs-reliquaires. Cette série de translations et de démembrements 3 translations, et au moins 4 démembrements - ne concerne que les saints du diocèse d'abord les martyrs puis également les saints abbés - et non ceux dont les corps ont été apportés de contrées lointaines à l'époque carolingienne. Elle semble, dès lors, traduire une exaltation de l'Église diocésaine, à laquelle les laïcs adhèrent au moins en partie. Dans ce processus, le tombeau du saint conserve une place privilégiée, même après l'élévation de ses reliques. Qu'il s'agisse d'un sarcophage ancien ou d'un monument tardif, il constitue un support fondamental de la mémoire des origines, un moyen de mettre en exergue l'ancienneté du culte.

La seconde moitié du XIV ${ }^{e}$ siècle, marquée par les guerres et l'apparition de la peste, connaît de nouveaux changements. Les pèlerins vénèrent de plus en plus les reliques fragmentaires de saints universels et, notamment, de saints thérapeutes, ainsi que des statues de la Vierge; ils fréquentent de plus en plus des églises paroissiales et des chapelles isolées. Le contexte paroissial regroupe ainsi 10 des 22 lieux de pèlerinage attestés au milieu du XV ${ }^{e}$ siècle. Malgré tout, les saints locaux ne sont pas en reste. Les laïcs témoignent à leur égard d'un intérêt certain qui se traduit notamment par la permanence ou la résurgence de certains pèlerinages - saint Didier de Langres, saint Bénigne de Dijon -, voire par la création de confréries en leur honneur dès le deuxième tiers du XIV ${ }^{e}$ siècle - confréries Saint-Didier à Langres et Saint-Robert à Molesmes. La fin du XV et le premier tiers du XVI ${ }^{\mathrm{e}}$ siècle voient même resurgir des cultes jusqu'alors 
peu documentés, comme celui de saint Valentin de Griselles ou de sainte Germaine de Bar-sur-Aube. De nouveaux reliquaires sont alors réalisés. À partir du second tiers du $\mathrm{XVI}^{\mathrm{e}}$ siècle, le culte des saints revêt des caractéristiques plus modernes que médiévales : l'imprimé constitue pour la première fois un moyen de promouvoir un pèlerinage (saint Vorles à Châtillon-sur-Seine), alors qu'apparaissent des sanctuaires sans reliques, bâtis notamment sur des fontaines - chapelle Saint-Gengoul à Varennessur-Amance ${ }^{6}$.

Dans le diocèse de Langres les saints sont demeurés des intercesseurs plus que des modèles. Le sanctoral local a conservé ses assises traditionnelles tout au long du Moyen Âge, ancrage que la forte implantation des bénédictins n'a fait que renforcer. La canonisation de Bernard de Fontaine est quant à elle restée marginale. Pour autant, les terres langroises n'offrent pas une image figée du culte des saints. En effet, au même titre que les chartes, la mémoire de leurs origines chrétiennes a fait l'objet d'interpolations et l'hagiographie de réécritures. L'intérêt des clercs et des laïcs à l'égard des saints locaux a par ailleurs fluctué, connaissant un nouvel essor alors que s'affirme, à la fin du Moyen Âge, un réel sentiment d'appartenance au diocèse.

\section{NOTES}

1. N. HERRMANN-MASCARD, Les reliques des saints. Formation coutumière d'un droit, Paris, 1975.

2. E. BOZOKY et A.-M. HELVÉTIUs (dir.), Les reliques. Objets, cultes, symboles, Turnhout, 1999.

3. PECIA, Ressources en médiévistique : Reliques et sainteté dans l'espace médiéval, $\mathrm{n}^{\text {os }} 8$-11, année 2005 et 2006.

4. L'inventaire est consultable en ligne : « http://www.coldev.org/sanctuaires ». 5. V. TABBAGH (dir.), Les clercs, les fidèles et les saints en Bourgogne médiévale (XI ${ }^{e_{-}}$ $\mathrm{XV}^{e}$ siècle), Dijon, 2005, p. 8.

6. Ce phénomène a été mis en évidence pour les diocèses d'Autun et de Nevers par Diane Carron. D. CARRON, Peuple de saints et pèlerinages dans les diocèses d'Autun et de Nevers, du temps des martyrs aux temps des réformes (IV ${ }^{\mathrm{e}}-\mathrm{XVIII}{ }^{\mathrm{e}}$ siècle), thèse de doctorat de l'université de Bourgogne sous la direction de Vincent Tabbagh, 2006. Résumé dans Bulletin du Centre d'études médiévales d'Auxerre, 11 (2007), [en ligne] http://cem.revues.org/document1108.html. 
INDEX

Index géographique : France/Langres

Mots-clés : saint, diocèse, corps saint 